\title{
BRUE: A new term and approach that could improve our practice
}

Throughout the history of medicine, there are many examples of definitions and terms associated with a specific approach or practice that have changed health care and have possibly improved results. For example, acute scrotum, acute surgical abdomen, and metabolic syndrome.

The term ALTE or apparent life-threatening event was established by expert consensus in 1986 and was intended to solve problems with the term "near-miss sudden infant death syndrome," which was used until then because there was no evidence relating it to sudden infant death syndrome (SIDS).

An ALTE may occur during sleep or a waking state. From an epidemiological perspective, it occurs at an earlier age than SIDS, i.e. one to three weeks earlier.

An ALTE was defined as an unexpected, sudden episode that is frightening and means impending or actual death to the observer. It is characterized by the combination of some of the following signs: respiratory pause, choking or gagging, color change (cyanosis, pallor, or florid complexion), and change in muscle tone.

One of the conclusions of an article published by us in 1995, last reviewed -to our delight- by Carlos Gianantonio, M.D., was that an ALTE did not account for a diagnosis in itself but simply referred to a clinical presentation. ${ }^{1}$

Acronym dissemination and its practical offshoots initially led to an improvement in diagnostic processes by differentiating, on one side, a mild event from a major one depending on the cardiovascular stimulation or resuscitation measures required to modify its course and, on the other side, according to the final diagnosis of idiopathic ALTE or ALTE secondary to various diseases. ${ }^{1-3}$

Over the years, different consensus meetings reinforced the need to take an adequate case history and do a careful physical exam to move forward with a selective testing schedule that is consistent with clinical experience and adequate for severe cases. ${ }^{3}$

However, many times medical practice has favored the performance of a series of diagnostic procedures, including patient hospitalization, although this may lead to an unnecessary risk, which on numerous occasions would probably fail to result in a treatable diagnosis.

The definition of ALTE caused uncertainty among health care providers because it assigned the event an inaccurate meaning by including the observer's subjective perception that it was lifethreatening or a feeling of impending death. Such perception resulted in a, many times, excessive health care and diagnostic response, rather based on a defensive attitude than on scientific knowledge, therefore evidencing how inadequate the term was.

The American Academy of Pediatrics (AAP) has recently published a new clinical practice guideline that recommended replacing the term ALTE with a new term: BRUE (brief resolved unexplained event). ${ }^{4}$

The authors define BRUE as an event occurring in infants younger than 1 year of age that is sudden and brief (lasting $<1$ minute), and resolves at the time of consult, characterized by at least one of the following signs: cyanosis or pallor, absent, decreased, or irregular breathing, marked change in muscle tone (hyper- or hypotonia), or altered level of responsiveness.

The current proposal also indicates that an event should be considered a BRUE only when there is no explanation after conducting an appropriate history and physical exam.

The AAP Subcommittee on ALTE performed a comprehensive review of the scientific literature from 1970 through 2014 to make the initial recommendations on BRUE management in infants.

Patients younger than 1 year old having a BRUE are classified as low-risk or high-risk patients based on their history and physical exam.

The guideline provides recommendations for BRUE with a low risk of recurrence and without an underlying condition which, in children who comply with the specific criteria, would allow a more conservative management. ${ }^{4}$

Based on this new definition, new guidelines or practice approaches will be agreed upon by scientific societies and in different health care settings for the management of children having these events. Such modification will possibly bring about a better quality, child- and familycentered medicine, together with a reduction in unnecessary and costly interventions.

Manuel Rocca Rivarola, M.D. Department of Pediatrics Department of Mother and Child Health Hospital Universitario Austral

http:/ /dx.doi.org/10.5546/aap.2016.eng.506 


\section{REFERENCES}

1. Rocca Rivarola M, Jenik A, Kenny P, Agosta G, et al. Evento de aparente amenaza a la vida. Experiencia de un enfoque pediátrico interdisciplinario. Arch Argent Pediatr 1995;93(2):85-91.

2. Kahn A, Rocca Rivarola M. ¿Qué es un evento de aparente amenaza a la vida (ALTE)? Arch Argent Pediatr 2001;99(1): 77-9.
3. Kahn A. Recommended clinical evaluation of infants with an apparent life-threatening event. Consensus document of the European Society for the Study and Prevention of Infant Death, 2003. Eur J Pediatr 2004;163(2):108-15.

4. Tieder JS, BonkowskyJL, Etzel RA, Franklin WH, etal. Brief Resolved Unexplained Events (Formerly Apparent LifeThreatening Events) and Evaluation of Lower-Risk Infants: executive summary. Pediatrics 2016;137(5):e20160591. 